\title{
ESTATÍSTICA E CLASSIFICAÇÃO DAS EMISSÕES SOLARES REGISTRADAS PELO CALLISTO-BR EM 2010 E 2011
}

\section{STATITICS AND CLASSIFICATION OF SOLAR EMISSIONS RECORDED BY THE CALLISTO-BR IN 2010 AND 2011}

\author{
José Declerk Buaca Sinadinse ${ }^{1}$ \\ Francisco Carlos Rocha Fernandes ${ }^{2}$ \\ Rafael Douglas Cunha-Silva ${ }^{3}$ \\ Zuleika Auxiliadora da Luz Sodré ${ }^{3}$
}

RESUMO: O CALLISTO-BR ("Compound Astronomical Low frequency Low Cost Instrument for Spectroscopy and Transportable Observatory"), em operação na Unidade Regional do INPE, em Cachoeira Paulista, SP, desde janeiro de 2010, é composto por dois espectrógrafos, nas polarizações horizontal e vertical. As observações são realizadas em ondas métricas (45 - $870 \mathrm{MHz}$ ), diariamente, das 12:00 UT às 22:00 UT. Entre março de 2010 e novembro de 2011, o CALLISTO-BR registrou 423 emissões solares. As emissões foram classificadas, de acordo com a morfologia apresentada, em emissões tipo I (2\%), tipo II (1\%), tipo III (50\%), tipo IV (< 1\%), tipo V (8\%) e emissões complexas ( 39\%). Neste trabalho, a estatística de ocorrência e a classificação dessas emissões são apresentadas.

Palavras-chave: CALLISTO-BR; emissões solares; ondas métricas; estatística.

ABSTRACT: The CALLISTO-BR (Compound Astronomical Low-cost Low-frequency Instrument for Spectroscopy and Transportable Observatory), in operation in the Regional Unit of INPE, at Cachoeira Paulista, SP, since January 2010, consists of two spectrographs, in the vertical and horizontal polarizations. The observations are made in metric wavelengths (45 - $870 \mathrm{MHz}$ ) daily from 12:00 UT to 22:00 UT. Between March 2010 and November 2011, the CALLISTO-BR recorded 423 solar emissions. The Emissions recorded were classified according to their morphology in emissions type I (2\%), type II (1\%), type III (50\%), type IV (<1\%), type V (8\%), and complex emission ( 39\%). In this work, the statistical occurrence and classification of these emissions are presented.

Keywords: CALLISTO-BR; solar emissions; metric wavelengths; statistics.

\footnotetext{
1 Graduando em Engenharia Elétrica - Universidade do Vale do Paraíba. E-mail: nucho-flo@hotmail.com.

2 Doutor em Astrofísica - INPE e Docente da Univap / Instituto de Pesquisa e Desenvolvimento - IP\&D. E-mail: guga@univap.br.

${ }^{3}$ Doutorando(a) em Física e Astronomia - Univap / IP\&D. E-mails: rfldoug@gmail.com; zuleika.sjc@bol.com.br. 


\section{INTRODUÇÃO}

As observações solares em comprimentos de onda métricos podem ser usadas como diagnóstico de vários processos físicos solares (MCLEAN; LABRUM, 1985). Os rádio-espectrógrafos para monitoramento da atividade solar, como o CALLISTO (BENZ; MONSTEIN; MEYER, 2005), podem contribuir para investigar tais fenômenos.

As rádio-emisões solares associadas aos fenômenos eruptivos solares, como flares e ejeções de massa coronal (CME), são classificadas em 5 tipos, de acordo com a morfologia apresentada nos espectros dinâmicos, descritos por McLean (1985) e apresentados, resumidamente, na Tabela 1.

Tabela 1 - Tipos de rádio-emissão registrados na faixa métrica

\begin{tabular}{|c|c|c|c|c|}
\hline Tipo & $\begin{array}{l}\Delta f \\
(\mathrm{MHz})\end{array}$ & $\begin{array}{l}\Delta \mathrm{t} \\
(\mathbf{s})\end{array}$ & Fonte de Energia & Mecanismo de emissão \\
\hline I & 4 & 1 & Dinâmica coronal & Turbulência de ondas de plasma \\
\hline II & $4-20$ & 300 & Ondas de choque & Turbulência choque \\
\hline III & 100 & 2 & Feixe de elétrons & Instabilidade bump on tail \\
\hline IV & 200 & 1000 & Não conclusivo & Instabilidade de cone de perda \\
\hline v & 30 & 20 & Instabilidade feixe & Não conclusivo \\
\hline
\end{tabular}

Vários catálogos e levantamentos de rádio-emissões solares, registradas em ondas métricas, foram publicados. Por exemplo, por Kundu et al. (1961), para a banda de frequência de 500 - $1000 \mathrm{MHz}$; por Dröge (1977), para a faixa de 200 - 1400 $\mathrm{MHz}$ e por Güdel e Benz (1988), para a banda de 300 - $1000 \mathrm{MHz}$. Mann et al. (1996) publicaram um catálogo de emissões do tipo II, registradas na faixa de 40 - 800 $\mathrm{MHz}$, e Bernold (1980) publicou um catálogo de estruturas finas de tipo IV de emissão, na faixa de 100 - $3000 \mathrm{MHz}$. Monstein (2011) apresenta uma catalogação de emissões registradas pelos espectrógrafos da rede eCallisto.

Neste trabalho, é apresentada a classificação e a estatística de ocorrência dos diferentes tipos de emissões solares registradas pelo CALLISTO-BR (SILVA; SELHORST; FERNANDES, 2010), na faixa de frequências métricas de 45 - $870 \mathrm{MHz}$, na fase de subida do ciclo solar 24. O CALLISTO-BR integra a rede global eCallisto (BENZ et al., 2009), atualmente com mais de 50 espectrógrafos em operação ao redor do mundo, que visa ao monitoramento contínuo da atividade solar na faixa de ondas métricas.

\section{OBSERVAÇÕES}

O CALLISTO-BR está instalado na Unidade Regional do INPE, em Cachoeira Paulista, SP. O instrumento é composto de dois espectrógrafos (nas polarizações horizontal e vertical) operando, em conjunto com duas antenas log-periódicas, na faixa de frequência de 45 a $870 \mathrm{MHz}$. As observações são realizadas, diariamente, das 12:00 UT às 22:00 UT. O CALLISTO-BR entrou em operação regular em 2010. Nos anos de 2010 e 2011, foram identificados 471 eventos com rádio-emissões solares 
métricas (SINADINSE et al., 2012), 2010 e de 2011 não houve observações conforme apresentado na Tabela 2. devido à manutenção e reparos no Ressalta-se que nos meses de setembro de instrumento.

Tabela 2 - Levantamento do número de dias de observação e do número de emissões registradas pelo CALLISTO-BR em 2010 e 2011

\begin{tabular}{lccc}
\hline Ano & $\begin{array}{c}\text { Dias de } \\
\text { observação }\end{array}$ & $\begin{array}{c}\text { Total de } \\
\text { eventos }\end{array}$ & Período investigado \\
\hline $\mathbf{2 0 1 0}$ & 218 & 144 & Março - Dezembro \\
$\mathbf{2 0 1 1}$ & 222 & 327 & Janeiro - Novembro \\
Total & 440 & 471 & \\
\hline
\end{tabular}

\section{CLASSIFICAÇÃO DAS EMISSÕES}

As rádio-emissões solares registradas pelo CALLISTRO-BR foram, então, preliminarmente catalogadas de acordo com sua morfologia apresentada no espectro dinâmico. A classificação levou em consideração características espectrotemporais, como: banda em frequência, duração, deriva em frequência, estruturas finas e forma. Cerca de $61 \%$ das emissões identificadas foram classificadas como um dos cinco tipos principais de emissão: tipo I (2\%), tipo II (1\%), tipo III (50\%), tipo IV (< $1 \%$ ) e tipo $V(8 \%)$ (Figura 1). Os $39 \%$ restantes foram agrupados em uma única categoria, especificada como "outros", pois apresentaram morfologia complexa, não permitindo uma classificação conclusiva apenas a partir das suas características espectro-temporais.

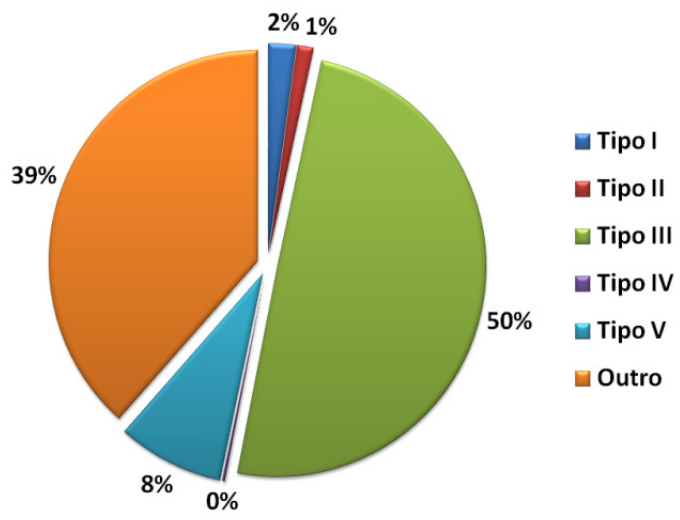

Figura 1 - Distribuição percentual das 471 rádio-emissões registradas pelo CALLISTO-BR no período de março de 2010 a novembro de 2011, classificadas de acordo com sua morfologia apresentada nos espectros dinâmicos. A categoria "outro" representa emissões com morfologia complexa, que não permitem uma classificação não ambígua e conclusiva.

Para ilustrar a grande variedade de emissões identificadas, exemplos são morfologias apresentada pelas rádio- mostrados nas Figuras 2 e 3. 


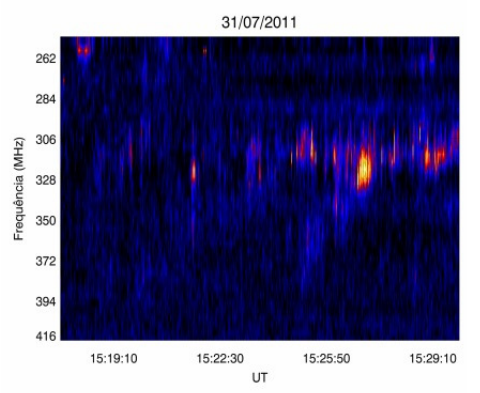

(a)

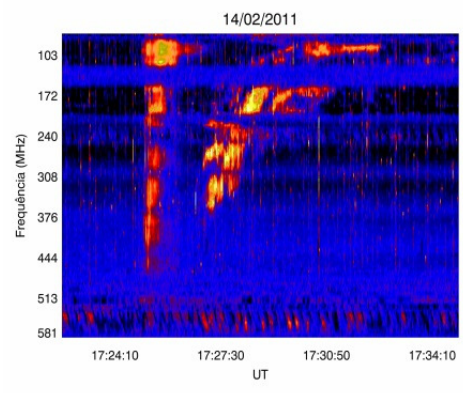

(d)

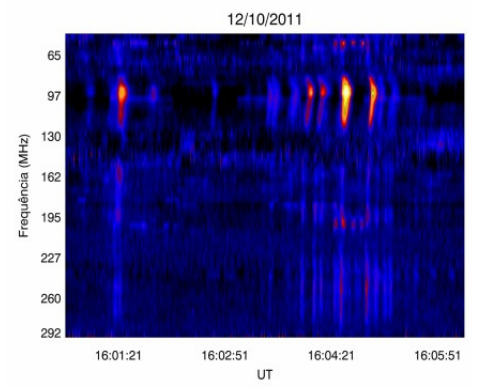

(g)

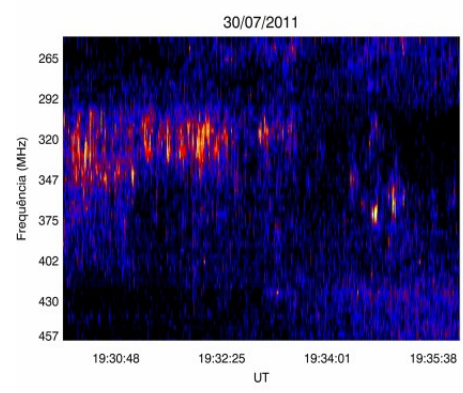

(b)

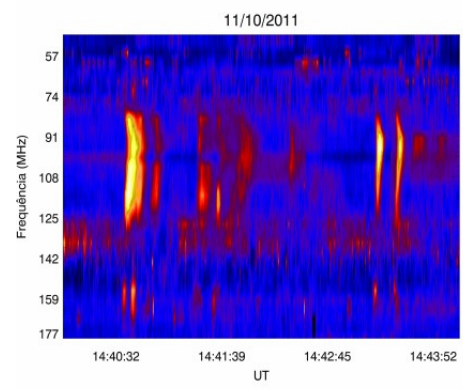

(e)

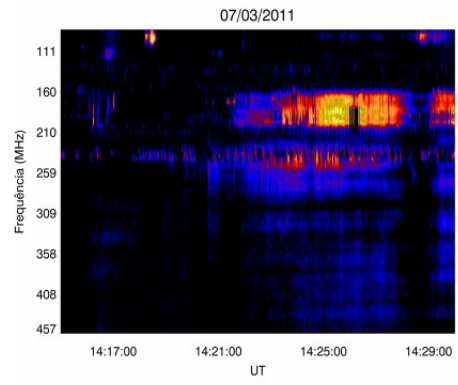

(h)

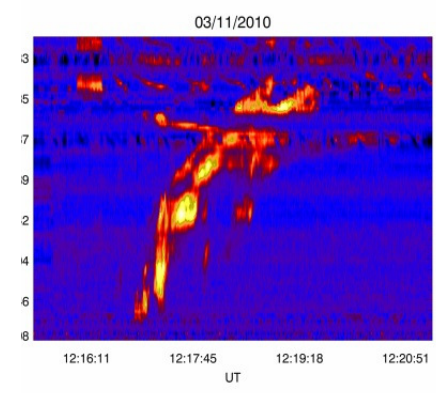

(c)

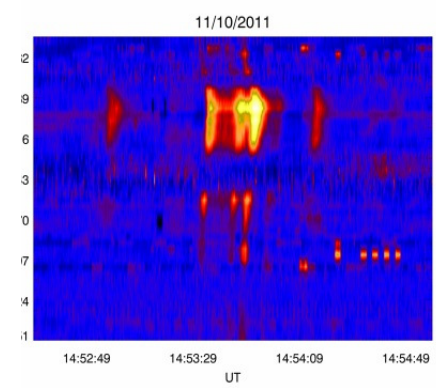

(f)

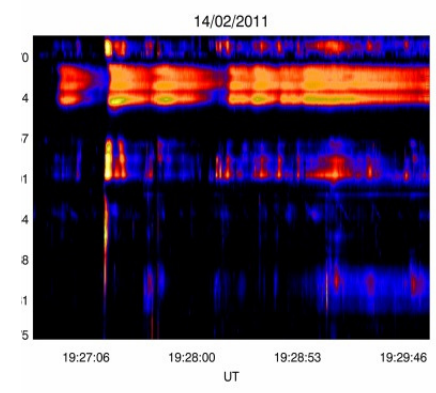

(i)

Figura 2 - Espectros dinâmicos de emissões típicas registradas pelo CALLISTO-BR: (a) e (b) correntes de emissões tipo I; (c) emissão tipo II com banda dividida; (d) emissão tipo II precedida por emissão tipo III; (e), (f) e (g) grupos de emissões tipo III; (h) emissão tipo IV; e (i) emissões tipo V precedidas por emissões tipo III.

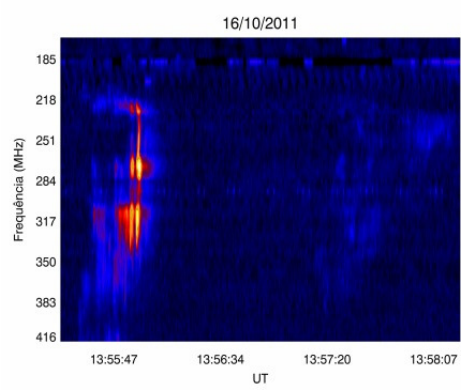

(a)

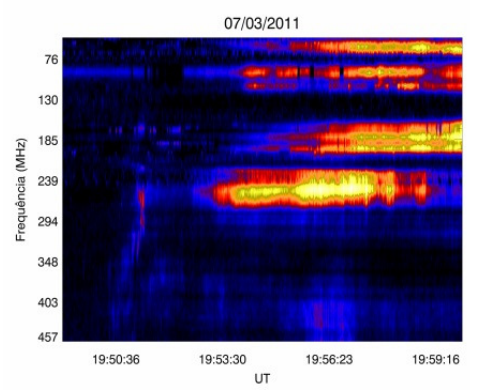

(b)

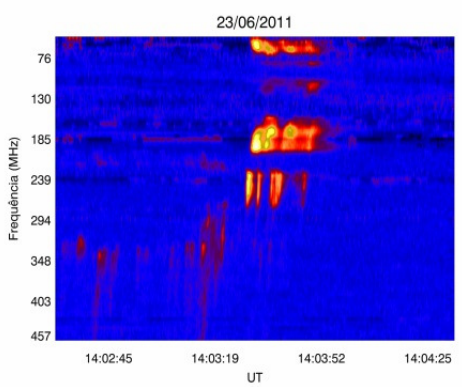

(c)

Figura 3 - Espectros dinâmicos de rádio-emissões solares com morfologia complexa preliminarmente classificadas como "outros tipos", registradas pelo CALLISTO-BR em: (a) 16/10/2011 ( 13:54 UT); (b) 07/03/2011 ( 19:50 UT); e (c) 23/06/2011 ( 14:01 UT). 


\section{CONCLUSÃO}

A principal contribuição de CALLISTO$\mathrm{BR}$, para o monitoramento contínuo da atividade solar na faixa métrica promovido pela rede e-CALLISTO, é a sua localização privilegiada, preenchendo uma lacuna observacional entre os instrumentos europeus e os norte-americanos.

Entre março de 2010 e novembro de 2011, foram identificadas quase 500 rádioemissões solares (45 - $870 \mathrm{MHz})$, registradas pela CALLISTO-BR. As emissões foram classificadas com base em sua morfologia espectral-temporal. Porém, para $38 \%$ das emissões registradas no período, não foi possível uma classificação conclusiva, seja por sua estrutura complexa ou à baixa intensidade, incluindo as emissões contínuas, estruturas pulsantes e outras morfologias. Atualmente, está em curso a classificação das emissões registradas em 2012.

As rádio-emissões identificadas estão sendo analisadas, com particular interesse na investigação de fenômenos solares associados com aceleração de partículas e ejeção e propagação de material coronal. Resultados preliminares das análises podem ser obtidos em Sodré, Cunha-Silva e Fernandes (2013) e Silva, Selhorst e Fernandes (2012).

\section{AGRADECIMENTOS}

J. D. B. Sinadinse agradece ao INCTAstrofísica/CNPq, pela bolsa de Iniciação Científica. R. D. Cunha-Silva agradece a bolsa de Doutorado - Processo $n^{\circ}$ 2012/00009-5, FAPESP e Z. A. L. Sodré agradece a Capes, pela bolsa de Doutorado. Ambos agradecem à Fundação Valeparaibana de Ensino, pela isenção de Revista Univap - Edição Especial - revista.univap.br São José dos Campos-SP-Brasil, v. 19, n. 34, nov.2013. ISSN 2237-1753 mensalidade. F. C. R. Fernandes agrade ao CNPq, pela Bolsa de Produtividade em Pesquisa (Proc. 308755/2012-0). Os autores agradecem ao EMBRACE-INPE, pelos dados do CALLISTO-BR.

\section{REFERÊNCIAS}

BENZ, A. O. Decimeter burst emission and particle acceleration. In: GARY, D. E., KELLER, C. U. (eds.) Solar and Space Weather Radiophysics. AA Dordrecht: Kluwer Academic Publishers. 2004. Chapter 10, p. 203-221.

BENZ, A. O.; MONSTEIN, C.; MEYER, H. CALLISTO - A New Concept for Solar Radio Spectrometers, Solar Physics, v. 226, p. 143-151, 2005.

BENZ, A. O. et al. A World-Wide Net of Solar Radio Spectrometers: e-CALLISTO, Earth, Moon and Planets, v. 104, p. 277-285, 2009.

BERNOLD, T. A catalogue of fine structures in type IV solar radio bursts. Astronomy and Astrophysics Supplement Series, v. 42, p. 43-58, 1980.

SILVA, R. D. C.; FERNANDES, F. C. R.; SELHORST, C. L. Physical Parameters of Shock-accelerated Electron Region

Estimated from Observations of Solar Metric Band-split Type II Burst. Physicæ Proceedings, Campinas, 1, mar. 2012. Disponível em: $<$ http://www.ifi.unicamp.br/physicae/ojs-

2.1.1/index.php/phyproceedings/article/view/ physicae.proceedings.XIYRM.5>. Acesso em: 06 nov. 2013.

DRÖGE, F. Millisecond fine-structures of solar burst radiation in the range 0.2-1.4 GHz. Astronomy and Astrophysics, v. 57, p. 285-290, 1977. 
GÜDEL, M.; BENZ, A. O. A catalogue of decimetric solar flare radio emission. Astronomy and Astrophysics Supplement Series, v. 75, p. 243-259, 1988.

KUNDU, M. R. et al. A comparison of the dynamic spectra of solar radio bursts in the decimeter and meter-wave-length ranges. Astrophysical Journal, v. 137, p. 572-579, 1961.

MANN, G. et al. Catalogue of solar type II radio bursts observed from September 1990 to December 1993 and their statistical analysis. Astronomy and Astrophysics Supplement, v. 119, p. 489-498, 1996.

MCLEAN, D. J.; LABRUM, N. R. Solar Radiophysics: Studies of Emission from the Sun at Metre Wavelengths. Cambridge: Cambridge University Press, 1985.

MONSTEIN, C. Catalog of dynamic electromagnetic spectra observed with Callisto. Physics, Astronomy and Electronics Work Bench. 2011. Disponível em: http://www.e-

callisto.org/papers/BurstCatalog.pdf. Acesso em: 05 jul. 2013.

SILVA, R. D. C.; SELHORST, C. L.; FERNANDES, F. C. R. O Espectrógrafo CALLISTO-BR e as Investigações de Emissões Solares em Ondas Métricas. In: ENCONTRO LATINO AMERICANO DE INICIAÇÃO CIENTÍFICA, 14., Encontro
LATINO AMERICANO DE PÓSGRADUAÇÃO (EPG), 10., ENCONTRO LATINO AMERICANO DE INICIAÇÃO CIENTÍFICA JUNIOR, 4., 2010, São José dos Campos, SP. Programação e anais de trabalhos completos. São José dos Campos: UniVap, 2010.

SINADINSE, J. D. B. et al. Estatística de emissões solares métricas $(45-870 \mathrm{MHz})$ observadas pelo CALLISTO-BR na fase de subida do ciclo solar 24. In: ENCONTRO LATINO AMERICANO DE INICIAÇÃO CIENTÍFICA, 16., ENCONTRO LATINO AMERICANO DE PÓS-GRADUAÇÃO (EPG), 12., ENCONTRO LATINO AMERICANO DE INICIAÇÃO CIENTÍFICA JUNIOR, 4., 2012, São José dos Campos, SP. Programação e anais de trabalhos completos. São José dos Campos: UniVap, 2012

SODRÉ, Z. A. L.; CUNHA-SILVA, R. D.; FERNANDES, F. C. R. Corrente de rádioemissões solares tipo I associada à tempestade de ruído observada em 14 de fevereiro de 2011. In: ENCONTRO LATINO AMERICANO DE INICIAÇÃO CIENTÍFICA, 16., ENCONTRO LATINO AMERICANO DE PÓS-GRADUAÇÃO (EPG), 12 ., ENCONTRO LATINO AMERICANO DE INICIAÇÃO CIENTÍFICA JUNIOR, 4., 2012, São José dos Campos, SP. Programação e anais de trabalhos completos. São José dos Campos: UniVap, 2012. 